\title{
Inflammatory myofibroblastic tumor of the endometrium mimicking a prolapsed fibroid
}

\author{
Mitchell B. Berger • Susan D. Ernst • \\ Richard W. Lieberman
}

Received: 5 May 2011 / Accepted: 31 May 2011 /Published online: 7 June 2011

(C) Springer-Verlag 2011

Keywords Inflammatory myofibroblastic tumor ALK1 . Prolapsed fibroid

\section{Introduction}

Inflammatory myofibroblastic tumors (IMTs) are rare neoplasms that were originally described in the lung, but have subsequently been reported in a variety of anatomic locations [1]. They were traditionally considered benign lesions, but are now classified as tumors of intermediate biological potential given a high likelihood of local recurrence and low incidence of metastasis [2]. While smooth muscle tumors (leiomyomata) are common in the uterus, IMTs are exceptionally rare, reported only in isolated case reports or case series [2,3]. We report a case of this unusual tumor arising from the endometrium but presenting like a prolapsed fibroid.

\section{Case presentation}

A 30-year-old gravida 4, para 3, Ab 1 presented to the emergency department with complaints of a 3-week history

M. B. Berger $(\bowtie) \cdot$ S. D. Ernst $\cdot$ R. W. Lieberman

Department of Obstetrics and Gynecology,

University of Michigan Health System,

L4000 Women's Hospital, 1500 East Medical

Center Drive, Room,

Ann Arbor, MI 48109-5276, USA

e-mail: mitcberg@umich.edu

R. W. Lieberman

Department of Pathology, University of Michigan Health System,

Ann Arbor, MI, USA of heavy vaginal bleeding. The bleeding started as light, continuous spotting for several days and then progressed to the point of requiring five to six pads per day. She had no other significant past medical or surgical history. Prior to this episode, she had regular menstrual cycles without abnormality. She denied a history of sexually transmitted infections or abnormal pap smears with a negative pap smear performed 6 months prior to the onset of this abnormal bleeding episode.

On pelvic examination, she was noted to have a $3-4-\mathrm{cm}$ mass protruding through the cervical os. The mass was round and smooth with multiple lobules and appeared white with a few small blood vessels coursing over the surface. It was firm, rubbery, and freely mobile growing off an approximately 5-mm-diameter stalk. The history and examination were felt to be consistent with a prolapsing fibroid, so the patient was counseled to undergo hysteroscopy and myomectomy.

The lesion was easily resected by placing it on traction, suture ligating the stalk, then transecting the stalk at its base with electrocautery. The mass weighed $9.5 \mathrm{~g}$, measured $3.5 \times 3.1 \times 2.8 \mathrm{~cm}$, and was found to be an inflammatory myofibroblastic tumor, with mesenchymal cells positive for desmin, alpha smooth muscle, and ALK 1 (Fig. 1).

\section{Discussion}

There have been a variety of pathologic lesions described using the term "inflammatory pseudotumor," including inflammatory myofibroblastic tumor, pseudosarcomatous myofibroblastic proliferations as well as malignant lesions [1]. However, it has recently been shown that IMT is a unique class of neoplasm. These masses are now known to 
Fig. 1 Photomicrograph demonstrating histologic appearance of the inflammatory myofibroblastic tumor. The appearance is of a spindle cell mesenchymal lesion (a; hematoxylin and eosin staining), with cells positive for ALK 1 (b), desmin (c), and alpha smooth muscle (d) staining
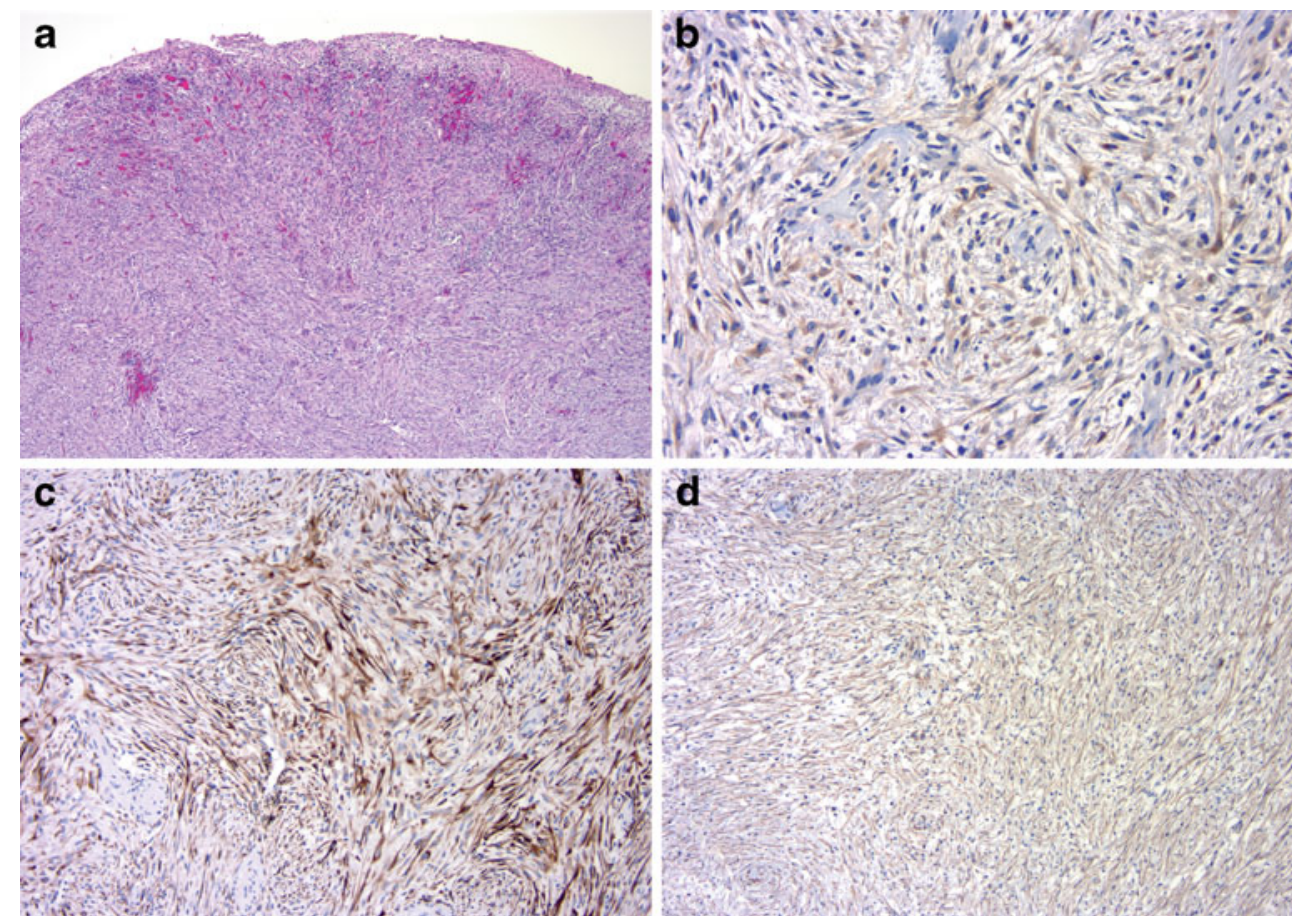

originate as a clonal population from a progenitor myofibroblast, have potential for local recurrence, invade locally or vascularly, and have potential for malignant transformation $[2,4,5]$.

IMTs are generally described as circumscribed or lobular lesions ranging from 1 to $20 \mathrm{~cm}$, with a mean size of $6 \mathrm{~cm}$, seen in various anatomic sites throughout the body $[1,6]$. Histologically, they are characterized by a triad of findings, including myofibroblastic proliferation, lymphoplasmacytic infiltrate, and myxoid background stroma [7]. They are most commonly found in children and adolescents, but can present in any decade of life. Some studies suggest that they are more commonly identified in men than women [6], although others suggest no gender preference [5]. Given that they have been identified in breast, urinary tract, and in the female pelvis and reproductive tract $[1,2]$, they may cause symptoms that lead a patient to present for evaluation to her gynecologist. For example, it has previously been reported that women with IMTs originating in reproductive organs may present with menorrhagia or abdominal discomfort [3, 7, 8]. In addition, IMTs may also be associated with vague systemic symptoms, such as malaise, fever, or weight loss $[1,4,6]$.

An important breakthrough in the ability to distinguish IMT from other lesions was the identification of rearrangements in the anaplastic lymphoma kinase $(A L K)$ locus on chromosome $2 \mathrm{p} 23$, leading to inappropriate expression of the ALK-1 protein [9]. This protein is normally only expressed in neural tissue, but has been found to be expressed in up to $70 \%$ of IMTs [1]. It has thus become a useful marker for immunohistochemical identification of IMT [3].

The first description of IMT was in the lung, where it is commonly found. However, several case reports and case series have documented the presence of these lesions in the female reproductive tract and pelvic cavity. It is critical that an IMT of the reproductive tract is examined histopathologically in order to distinguish it from other inflammatory, malignant, or benign processes. For example, syphilis has been reported to present as presumed lymphosarcoma of the cervix [10]. In addition, IMTs can grossly appear similar to benign masses like fibroids or malignant tumors such as uterine leiomyosarcomas. To the best of our knowledge, there are only two cases in a single case series of an endometrial IMT protruding through the cervical os, mimicking a prolapsed fibroid, reported to date [7].

IMT is now described as a neoplasm of intermediate biological potential due to its ability to recur, invade locally, or metastasize $[1,5,8]$. Surgical extirpation is the mainstay of therapy, although chemotherapeutic agents and radiation therapy have also been used with some success [2, 5]. Given the rarity of these tumors, there are no standardized guidelines for management. However, case series suggest that aggressive, radical surgical extirpation is unwarranted. Rather, close observation or local resection, e.g., hysteroscopic removal or simple hysterectomy, should be offered [6-8].

Based on the risk for recurrence or metastasis and the fact that our patient expressed that she had completed childbearing, she was offered observation with annual 
imaging or hysterectomy. She opted for conservative management and, at 1 year postoperatively, has been asymptomatic with normal-appearing uterine and endometrial contour and no adnexal masses on pelvic ultrasound.

\section{Conclusion}

Inflammatory myofibroblastic tumors of the endometrium may mimic other benign or malignant gynecologic pathologies. Although these tumors are rare, they do have intermediate biological potential. As such, it is critical that accurate histologic diagnosis of any resected lesion be obtained in order to appropriately counsel a patient about future follow-up and possible long-term sequelae.

Conflicts of interest The authors report no conflicts of interest. The authors alone are responsible for the content and writing of this paper.

\section{References}

1. Gleason BC, Hornick JL (2008) Inflammatory myofibroblastic tumours: where are we now? J Clin Pathol 61:428-437
2. Kovach SJ, Fischer AC, Katzman PJ, Salloum RM, Ettinghausen SE, Madeb R, Koniaris LG (2006) Inflammatory myofibroblastic tumors. J Surg Oncol 94:385-391

3. Shintaku M, Fukushima A (2006) Inflammatory myofibroblastic tumor of the uterus with prominent myxoid change. Pathol Int 56:625-628

4. Azuno Y, Yaga K, Suehiro Y, Ariyama S, Oga A (2003) Inflammatory myoblastic tumor of the uterus and interleukin-6. Am J Obstet Gynecol 189:890-891

5. Coffin CM, Hornick JL, Fletcher CD (2007) Inflammatory myofibroblastic tumor: comparison of clinicopathologic, histologic, and immunohistochemical features including ALK expression in atypical and aggressive cases. Am J Surg Pathol 31:509-520

6. Cheng L, Foster SR, MacLennan GT, Lopez-Beltran A, Zhang S, Montironi R (2008) Inflammatory myofibroblastic tumors of the genitourinary tract-single entity or continuum? J Urol 180:12351240

7. Rabban JT, Zaloudek CJ, Shekitka KM, Tavassoli FA (2005) Inflammatory myofibroblastic tumor of the uterus: a clinicopathologic study of 6 cases emphasizing distinction from aggressive mesenchymal tumors. Am J Surg Pathol 29:1348-1355

8. Gucer F, Altaner S, Mulayim N, Yapicier O (2005) Invasive inflammatory pseudotumor of uterine cervix: a case report. Gynecol Oncol 98:325-328

9. Dehner LP (2004) Inflammatory myofibroblastic tumor: the continued definition of one type of so-called inflammatory pseudotumor. Am J Surg Pathol 28:1652-1654

10. Drusin LM, Singer C, Valenti AJ, Armstrong D (1977) Infectious syphilis mimicking neoplastic disease. Arch Intern Med 137:156160 